\title{
29 \\ BOARD MEETINGS AND THE AGENDA
}

\author{
Dineshi Ramesh
}

The board's remit is vast, yet it has limited time at its disposal. The best board meetings move an organisation closer towards its goals whilst giving directors confidence that they are discharging their duties well.

Effective board meetings do not happen by chance. There is extensive planning behind the scenes, and a careful balance needs to be struck between structuring the meeting well and human interaction. A great conversation is what you're aiming for, as Theodore Zeldin ${ }^{1}$ explains,

When minds meet, they don't just exchange facts: they draw different implications from them; engage in new trains of thought. Conversation doesn't just reshuffle the cards; it creates new cards.

\section{The challenge}

The challenge is how to ensure the board meeting focuses on what matters most. What are the conversations the board needs to be having and how do

1 Conversation: How Talk Can Change Our Lives Theodore Zeldin, Mahwah, NJ: HiddenSpring, (C2000.

DOI: $10.4324 / 9781003201182-35$ 
you ensure vital topics do not get crowded out? Board intelligence in collaboration with the Cambridge Judge Business School conducted research that shows that boards typically spend too much time in one of two areas: governance (a pronounced concern in financial services) and in the "weeds" of operational performance.

Most boards say they want to be spending more time on strategy and looking at how the organisation is progressing towards its long-term goals. The reality can be weighed down by firefighting or operational issues. By designing a forward-looking calendar built around the board's priorities, the balance can be redressed.

\section{Purpose and the agenda}

The foundation of an effective board meeting is the agenda. Most chairs design their board agenda in consultation with their company secretary and chief executive. The starting point tends to be the list of "must do" items - be they statutory, administrative, or routine in nature. They are easy to recall, and they often fall into the "boring but important" category of meeting items.

However, the board agenda should start with the important topics, which are sometimes harder to identify. Figuring out the conversations the board should be having is the starting point for any good agenda. Let's look at this in three steps.

\section{Step 1 - what are the board's priorities?}

A good starting point is to answer two essential questions for the coming 12-18 months:

- What are the big decisions this forum will take or shape (steering)?

- What are the big items this forum should monitor (supervising)?

To ensure you cover the right bases, consider these two questions across three areas: strategy, performance, and governance. Try to phrase your responses in the form of questions to help drive specificity and avoid ambiguity. For example, rather than an item on "culture," be specific: "how well have we embedded our culture?" Rather than an agenda 
item on "growth," specify "what is the right mix of acquisitive and organic growth for 202x?"

By posing these issues as questions, it becomes clear whether the style of conversation being prompted is steering or supervising in nature. This in turn, signals to the board to get ready to make a decision or to challenge performance.

By agreeing the board's priorities, there will be:

- A shared sense of purpose - the board will be united in their understanding of the issues that matter most in the coming year. This reduces the risk of misaligned expectations between members and makes it easier to chair the forum.

- Clarity for the executive - the remit of the board on each topic is clear. The executive can decide how to prepare great information to support the board's priorities and if there are other priorities that should remain the preserve of management.

\section{Step 2 - how to construct the forward calendar?}

Translating these priorities into the forward calendar is the next step in shaping the board agenda. Consider the appropriate timing or frequency:

- When should the steering priorities come to the board? Allocate a month.

- How often should the supervising priorities come to the board? For example, monthly, quarterly, or just the once. Supervising is about providing assurance that activity is on track, so the topic may need to come to the board more than once.

In practice, there are other considerations, especially if the organisation's governance structure is more complex than a single board and executive committee. The key here is to coordinate the board calendar with the governance structure it operates within. Once you've done this, you'll have an outline of the board's forward calendar of meetings. 


\section{Step 3 - how can you design an agenda that drives a better conversation?}

The challenge is how to make best use of everyone's time: the order of matters, the time given to each item, how to regulate energy and concentration, and how to make it is easy for participants to switch between different modes of thinking.

Here are some of the hallmarks of a great agenda:

I. Open the meeting with the chair's remarks and the CEO's report, rather than an administrative item, for example, the minutes. This sets the scene.

II. Allow for fewer but deeper conversations about the most important topics. This is what board members prefer; time to get to the heart of the matter.

III. Group items that require similar modes of thinking together - steering (decision-making) versus supervising (monitoring). Cognitively, this reduces strain.

IV. Consider "zero timing" items. A neat way of dealing with papers that require no discussion, they are purely for note and need not consume agenda time.

V. Build in ample breaks. A tired board is not an effective one.

Now the foundation of the meeting is in place (its purpose and agenda). Let's look at the other drivers of a great meeting.

\section{Meeting effectiveness}

One of the most effective ways of helping boards improve their meetings is to self-assess how well the meeting is working (or not!). Unpacking the drivers of a great meeting improves the board's understanding of what it takes to have a more effective meeting. Repeating the exercise will prompt changes to be made from one meeting to the next. Here are five drivers to consider:

- Purpose and Agenda - covered above

- Information and security 
- $\quad$ People and place

- Behaviours

- Decisions and progress

Within each driver there is a series of questions that help demystify what makes for a great meeting. ${ }^{2}$

\section{Questions to ask \\ Purpose and agenda}

- Is the purpose of the forum clear?

- Is meeting time being spent on the right topics?

- Do we have the right number of meetings to discharge our duties?

- Is there a forward calendar outlining the forum's priorities?

- Is the agenda well structured with clarity on items for decision versus supervision?

\section{Information and security}

- Is high-quality information provided as a pre-read?

- Are there systems and processes in place to support secure, electronic distribution of papers that facilitate collaboration?

- Is the "ask" of the reader clear for each paper?

- Are papers unvarnished, forward-looking, and insightful?

- Is information quality-assured by sponsors with audit trails for key decisions?

\section{People and Place}

- Do members represent ample diversity of thought?

- Do we have the right number of members with the required skills (not too many/few)?

- Does the physical set-up and timing support participants' energy?

- Do members adequately represent our key stakeholders?

2 Smart Meeting Score (C) Board Intelligence. 
- Does the set-up of the meeting (physical or virtual) facilitate collaboration (pre- and during the meeting)?

\section{Behaviours}

- Does the chair encourage an atmosphere of trust?

- Does the forum contribute effectively (making decisions where required, providing challenge/support depending on under-/ overperformance)?

- Is the meeting chaired effectively (kept on agenda/to time)?

- Are conversations constructive (open-minded, upholding the forum's purpose)?

- Does the discussion build on thinking (injecting ideas, solutions) rather than being disjointed/inconclusive?

\section{Decisions and progress}

- Does the meeting drive the organisation forward?

- Do we seek to improve our meetings?

- How effective is decision-making?

- Do decisions consider the impact on the long term, the environment, and all relevant stakeholders?

- Are attempts made to ensure decision-making is not skewed by cognitive bias?

These questions have been tested in the field through observation of real board and executive committee meetings, and though not exhaustive, they tend to be the ones that move the dial when it comes to making meetings better. The questions are most powerful when answered by the board as a whole and the results amalgamated. If completed anonymously, you'll get the truest picture of what your board really thinks. Use the results as a calibration point from which you can begin to have better meetings, better conversations, and ultimately move both your organisation and its role in society forward, in the most positive way. 